Themenheft Nr. 46: Eltern - Pädagog*innen - Medienkompetenzen.

Eltern und Pädagog*innen zwischen Aneignung und Vermittlung von Medienkompetenz. Herausgegeben von Thorsten Naab, Alexandra Langmeyer, Ruth Wendt und Jessica Kühn

\title{
sIch habe eigentlich das Gefühl, so, wie es jetzt im Augenblick läuft, wird diese Lerntätigkeit auf die Eltern abgewälzt)
}

Eine figurationsanalytische Betrachtung von Familien während der ersten Phase der Covid-19-Pandemie

Paul Petschner ${ }^{1}$, Andreas Dertinger ${ }^{1}$ (D), Claudia Lampert ${ }^{2}$ (D) und Jane Müller ${ }^{1}$ (D)

${ }^{1}$ Friedrich-Alexander-Universität Erlangen-Nürnberg

${ }^{2}$ Leibniz-Institut für Medienforschung | Hans-Bredow-Institut (HBI)

\begin{abstract}
Zusammenfassung
Im März 2020 kam es aufgrund der Covid-19-Pandemie zu landesweiten Schulschliessungen. Lehrkräfte, Eltern und deren schulpflichtige Kinder wurden von dieser Situation völlig unvorbereitet getroffen. Der Beitrag beleuchtet diese erste Phase des Distance Schooling mit Blick auf die damit einhergehenden Anforderungen an Familien und veränderte familiale Interaktionsprozesse. Unter Fokussierung auf medienbezogene Aushandlungsprozesse und veränderte Konstellationen von Medienpraktiken werden die Ergebnisse einer qualitativen Erhebung mit sechs Familien eines bestehenden Längsschnitt-Panels vorgestellt. Zum Erhebungszeitpunkt im April/Mai 2020 befanden sich die Kinder in der sechsten Jahrgangsstufe. Die Interaktionsprozesse werden ausgehend von dem Ansatz kommunikativer Figurationen beschrieben, wobei der Fokus auffamiliale Aushandlungsprozesse im Spannungsfeld von Autonomie und Heteronomie gelegt wird. Die Ergebnisse veranschaulichen, mit welchen Herausforderungen die (Ausnahme-)Situation des Distance Schooling verbunden ist und wie heterogen Familien mit diesen umgehen. In der familialen Interaktion ergeben sich neue Freiheitsgrade und Anforderungen an ein eigenständiges Handeln der Kinder, die innerhalb der Familie ausgehandelt und bewältigt werden müssen.
\end{abstract}


«I Actually Have the Feeling, Given the Current Circumstances That the Learning Activities Are Shifted over to the Parents). A Figurational-Analysing Consideration of Families during the First Phase of the Covid-19 Pandemic

\begin{abstract}
Due to the Covid-19 pandemic schools were closed state-wide in March 2020. Teachers, parents and school-age children were completely unprepared for this situation. This article examines the first period of Distance Schooling with regard to the concomitant requirements to families and changing family interactions. The findings of a qualitative survey with six families of an existent longitudinal panel are introduced, focussing on media-based negotiation processes and changing constellations of media practices. At the time of the survey in April and May 2020, the children were stated in the 6th grade. The interaction processes will be developed based on the concept of communicative figurations, though the focus is placed on family negotiation processes in the field of tension between autonomy and heteronomy. The findings illustrate the attendant challenges of the (exceptional) circumstances of Distance Schooling and the heterogeneous ways families handle them. Family interaction produces new extents of freedom and requirements to independent actions of children, which need to be negotiated and mastered within the family.
\end{abstract}

\title{
1. Veränderungen durch Distance Schooling für Familien
}

Die Covid-19-Pandemie stellte die Bevölkerung vor bisher unbekannte Herausforderungen. In Deutschland wurden die ersten einschneidenden politischen Massnahmen zur Eindämmung des Infektionsgeschehens im Frühjahr 2020 verabschiedet. Mitte März vereinbarten die Bundesregierung und die Regierungschefinnen und -chefs der Bundesländer erstmals Leitlinien zur Beschränkung sozialer Kontakte im öffentlichen Raum (BPA 2020a; 2020b). Zwischen dem 16. und 18. März traten in allen Bundesländern temporäre Schulschliessungen in Kraft (Fickermann und Edelstein 2020). Kontaktbeschränkungen, die damit einhergehende Eindämmung von Freizeitaktivitäten sowie der abrupte Wegfall des formal-institutionalisierten Rahmens von Schule stellten eine Ausnahmesituation dar, die insbesondere für Familien und schulpflichtige Kinder einen massiven Einschnitt in den Alltag bedeutete (Andresen u. a. 2020; Langmeyer u. a. 2020). Eltern und deren schulpflichtige Kinder wurden völlig unvorbereitet von den ersten pandemiebezogenen Regelungen getroffen, die u. a. die Aufrechterhaltung des Schulbetriebs in Form von Distance Schooling umfassten. Zu einem Zeitpunkt, als viele Eltern auf unterschiedliche Weise von Einschnitten in ihrem Berufsalltag betroffen waren (Möhring u. a. 2020), mussten Familien zusätzlich ihre Organisation schulischen Lernens restrukturieren (Gerhardts u. a. 2020a; 
2020b). Für die Bewältigung der pandemiebedingten Einschränkungen wurden digitale Informations- und Kommunikationsmedien nahezu unverzichtbar. Dabei trafen die neuen Anforderungen auf domänenspezifische medienbezogene Ressourcen in den Familien.

Erste Hinweise auf eine veränderte Rolle von Medien im familialen Kontext liefern mehrere quantitative Surveys, die im Anschluss an die ersten Schulschliessungen im April 2020 erhoben wurden. Wie aus den Befragungen hervorgeht, erwiesen sich EMails als zentrales Kommunikationsmedium zwischen Lehrkräften und Familien. Der Mailverkehr diente hauptsächlich der Übermittlung von Arbeitsaufträgen, wohingegen ein direkter Austausch zwischen Lehrkräften und den Schülerinnen und Schülern (z. B. via Videokonferenzen) seltener realisiert wurde (Heller und Zügel 2020; JIMplus 2020; Lampert, Thiel, und Güngör 2021; Langmeyer u. a. 2020; Robert-BoschStiftung 2020; Vodafone Stiftung 2020; Wacker, Unger, und Rey 2020; Wildemann und Hosenfeld 2020; Wößmann u. a. 2020). ${ }^{1}$ Hauptunterstützende der Lerntätigkeiten von Kindern im Distance Schooling waren deren Eltern (Heller und Zügel 2020; JIMplus 2020). Die am häufigsten für das Distance Schooling benötigten technischen Geräte waren Smartphones, Computer oder Laptops sowie Drucker, wobei nahezu alle befragten Schülerinnen und Schüler angaben, über die entsprechenden Geräte zu verfügen (Heller und Zügel 2020; Huber u. a. 2020; JIMplus 2020). Während des Distance Schooling zeigte sich eine quantitative Zunahme der Nutzungsdauer digitaler Medienangebote durch Heranwachsende (JIMplus 2020; Lampert, Thiel, und Güngör 2021; Langmeyer u. a. 2020; Wößmann u. a. 2020).

Die durch die ersten Schulschliessungen bedingten Veränderungen des Familienalltags erlebten und bewerteten Familien sehr unterschiedlich. Einige Studien zeichnen ein insgesamt positives Bild. In einer JIMplus-Erhebung bewerteten $84 \%$ der befragten Schülerinnen und Schüler den Unterricht zu Hause mindestens mit der Schulnote drei, 52\% davon vergaben die Note eins oder zwei (JIMplus 2020). Auch Eltern gaben mehrheitlich an, mit der Organisation der Schularbeiten ihrer Kinder (Vodafone Stiftung 2020) sowie der Gesamtsituation während der Schulschliessungen (Wößmann u. a. 2020) gut zurechtgekommen zu sein. Es wurde aber auch von Problemen des Distance Schooling berichtet. Hierzu zählten u. a. die Schwierigkeit, geringe zeitliche Kapazitäten und fehlende Kenntnisse mit gestiegenen Unterstützungsbedarfen der Kinder zu vereinbaren oder der Wunsch nach einer besseren Organisation und Unterstützung von Schulen und Lehrkräften (Vodafone Stiftung 2020). Noch deutlicher zeigte sich das differente Erleben des Distance Schooling in den sehr heterogenen Antworten einzelner Schülerinnen und Schüler sowie Eltern auf offene Fragen zur Wahrnehmung der familialen Gesamtsituation (Andresen u. a.

1 Gerhardts u. a. (2020a, 15) differenzieren die erste Phase des Distance Schooling in zwei Zeiträume: die «Ad-hoc-Phase» vom Beginn der Schulschliessungen bis zu den Osterferien und eine «strukturierte Phase» nach den Osterferien 2020, in der auch zunehmend synchrone Lehrformate angeboten wurden. 
2020) und der Bewertung des Distance Schooling (Heller und Zügel 2020). Mal wurden katastrophale Umstände beschrieben, mal überwogen einzelne negative oder positive Aspekte oder es wurden ausschliesslich positive Effekte betont.

Insgesamt zeigen die vorliegenden Studien konsistente Befunde bezüglich der Mediennutzung und der Situation von Familien im Anschluss an die ersten Schulschliessungen im Frühjahr 2020. Vertiefender Forschungsbedarf besteht dabei hinsichtlich der Qualität der veränderten Mediennutzung sowie der Ursachen und Hintergründe der heterogenen Wahrnehmung der Umstände durch die Familien. Der vorliegende Beitrag setzt hier an und nimmt die Wahrnehmungen von Eltern und Kindern sowie das familiale Beziehungsgeflecht aus einer figurationsanalytischen Perspektive genauer in den Blick.

\section{Distance Schooling aus figurationsanalytischer Perspektive}

Unabhängig von dem Einfluss der Covid-19-Pandemie ist unsere Gesellschaft von Veränderungsprozessen geprägt, die mit dem Begriff der «tiefgreifenden Mediatisierung» beschrieben werden (Hepp 2020). Durch diesen Metaprozess verändern sich kommunikative Praktiken und hiervon ausgehend die gesellschaftliche Konstruktion der Wirklichkeit. Die Effekte konkretisieren sich auf der Meso-Ebene sozialer Domänen (Hepp und Hasebrink 2017, 332-334). Die mediengestützte Kommunikation ist hierbei ein konstitutiver Faktor der jeweiligen Domäne. Der Ansatz kommunikativer Figurationen (Hepp und Hasebrink 2014; 2017) bietet ein geeignetes Instrument zur Erfassung medienbezogener Transformationsprozesse innerhalb verschiedener sozialer Domänen. Dieses Konzept schliesst an den Figurationsbegriff von Elias (1970) an, mit dem Ziel, die Auswirkungen der tiefgreifenden Mediatisierung auf der Ebene sozialer Domänen zu untersuchen. In ihrer Handlungspraxis sind die Akteurinnen und Akteure der Figuration miteinander verwoben, weshalb diese mit ihren interdependenten Praktiken eine gemeinsame Wirklichkeit konstruieren (Elias 1970). Ausgehend von diesem Verständnis kann die konventionelle Trennung zwischen Individuum und Gesellschaft kritisch betrachtet und relativiert werden (Elias 1970, 139). Figurationen sind u. a. durch «fluktuierende Machtbalancen» (Elias 1970, 143) und emotionale Bezogenheiten (Valenzen) (Elias 1970, 147) zwischen den Agierenden geprägt. Als dynamische Gebilde verändern sich Figurationen (und damit auch Machtverhältnisse und Valenzen) kontinuierlich.

Durch die Ausdifferenzierung des Aspektes medial vermittelter Kommunikation im Konzept der kommunikativen Figurationen wird Elias Figurationsansatz von Hepp und Hasebrink $(2014$; 2017) für kommunikationswissenschaftliche Forschungen erweitert. Als kommunikative Figurationen werden die kommunikativen Interdependenzgeflechte einer Akteurskonstellation verstanden, die über ein geteiltes Medienensemble hinweg bestehen und durch eine spezifische Thematik gerahmt sind 
(Hasebrink 2014, 227). Innerhalb der kommunikativen Figuration kann zwischen dem Medienensemble und dem Medienrepertoire unterschieden werden. Das Medienensemble umfasst alle innerhalb der sozialen Domäne vorhandenen Medien. Das Medienrepertoire ist akteursspezifisch und bezieht sich auf die individuellen Medien(praktiken) einer Akteurin oder eines Akteurs innerhalb der sozialen Domäne. Der Ansatz der kommunikativen Figurationen bildet zudem eine adäquate Grundlage zur Beschreibung mediatisierter familialer (Sozialisations-)Prozesse (Kammerl u. a. 2020; 2021). Dabei rückt insbesondere die Akteurskonstellation zwischen Kind und Eltern in den Fokus. Für die Ausgestaltung der Machtbalancen zwischen Kind und Eltern(teil) sind Aushandlungsprozesse, welche die Autonomie und Heteronomie des Kindes betreffen, von Bedeutung (Kammerl u. a. 2021, 195).

Die abrupte Umstellung des schulischen Lernens in der ersten Phase des Distance Schooling wird im Folgenden als eine Irritation konzipiert, die das Potenzial beinhaltet, sich auf die bestehenden Strukturen der kommunikativen Figuration Familie auszuwirken. In den Familien müssen Wege und Routinen gefunden werden, den Kindern schulisches Lernen unter den veränderten Bedingungen zu ermöglichen (Gerhardts u. a. 2020a; 2020b). Insbesondere durch die (für die erste Phase des Distance Schooling konstitutive) primär asynchrone Bereitstellung von Aufgaben (Unger, Krämer, und Wacker 2020, 86) wurden Familien vor zusätzliche Herausforderungen hinsichtlich der Verantwortlichkeiten bei der Organisation von Schularbeiten gestellt (Gerhardts u. a. 2020b, 144). Lehrkräfte fielen somit für die Klärung von Rückfragen und die Prüfung der Schulkinder weg. Gleichzeitig wurde durch den Entfall des Präsenzunterrichts von den Kindern eine gestiegene Eigenverantwortung hinsichtlich des Abrufs, der Organisation und Bearbeitung der Arbeitsaufträge verlangt. Diese neue Konstellation der familialen Lernorganisation kann potenziell Aushandlungsprozesse um Autonomie und Heteronomie zwischen Kind und Eltern(teil) hervorrufen. Veränderungen betreffen zudem das Medienrepertoire des Kindes, da schulisches Lernen im Distance Schooling an technische Endgeräte geknüpft ist. Die Kinder sind so auf veränderte Mediennutzungsformen und -zeiten angewiesen und müssen eventuell auf neue Endgeräte zugreifen. Hierdurch werden möglicherweise auch medienerzieherische Praktiken innerhalb der Familie tangiert. Im Folgenden richtet sich der Blick auf die Aushandlung der Machtbalance in der Akteurskonstellation zwischen Kind und Eltern(teil), die durch die Umstände während des Distance Schooling herausgefordert wurde, sowie auf Veränderungen im Medienrepertoire der Kinder. 


\section{Studiendesign und Fragestellungen}

Die explorative Studie basiert auf der Befragung von sechs Familien, die Teil eines bestehenden Längsschnittpanels ${ }^{2}$ sind. Zum Erhebungszeitpunkt (April/Mai 2020) befanden sich die Kinder in der sechsten Jahrgangsstufe. Aufgrund der geltenden Kontaktbeschränkungen fanden die Befragungen per Videotelefonie statt, die Audiospur wurde dabei aufgezeichnet. In jeder Familie wurden mit leitfadengestützten Interviews getrennte Gespräche mit dem Kind und jeweils einem Elternteil geführt. Die Auswertung der Interviewtranskripte erfolgte mit der qualitativen Inhaltsanalyse nach Kuckartz (2020) unter Verwendung der Software MAXQDA 2020. Die Daten wurden mit einem hierarchischen Kategoriensystem analysiert, dessen Oberkategorien theoriegestützt deduktiv entwickelt und deren Unterkategorien induktiv am Material abgeleitet wurden.

Alle sechs Familien leben in abgesicherten sozioökonomischen Verhältnissen im Einzugsgebiet oder direkt in einer Grossstadt. In zwei Fällen leben die Eltern getrennt. Lisas Mutter wohnt mit einem neuen Lebenspartner zusammen, Hannahs Mutter ist alleinerziehend. Fünf Kinder «besuchen» ein Gymnasium, eine Schülerin eine Realschule. Das Sample weist ein deutliches Bias auf. Bereits im Rekrutierungsprozess des bestehenden Längsschnitt-Panels konnten trotz vielfältiger Bemühungen primär Familien aus einem vorwiegend «bürgerlichen» Milieu für die Studienteilnahme gewonnen werden.

\begin{tabular}{|l|l|l|}
\hline Pseudonym & Schulform & befragter Elternteil, Bildungsabschluss \\
\hline Tobias & Gymnasium & Vater, Realschulabschluss \\
\hline Jakob & Gymnasium & Mutter, Realschulabschluss \\
\hline Johann & Gymnasium & Mutter, Realschulabschluss \\
\hline Lisa & Realschule & Mutter, Hochschulabschluss \\
\hline Pia & Gymnasium & Mutter, Hochschulabschluss \\
\hline Hannah & Gymnasium & Mutter, Realschulabschluss \\
\hline
\end{tabular}

Tab. 1.: Überblick über das Sample.

Die folgenden beiden Forschungsfragen bilden die Grundlage der in diesem Beitrag elaborierten Ergebnisse:

- Wie verändern sich das familiale Medienensemble und das kindliche Medienrepertoire durch die Situation während des Distance Schooling?

- Welche autonomiebezogenen Aushandlungsprozesse in der Akteurskonstellation zwischen Kind und Eltern(teil) mit Bezug auf das Distance Schooling zeigen sich in der kommunikativen Figuration Familie?

2 Hierbei handelt es sich um das qualitative Panel des DFG-Projekts «Connected Kids - Sozialisation in einer sich wandelnden Medienumgebung (Conkids)» (KA 1611/ LA 2728, Laufzeit: 2018-2021). 
Mit diesen Forschungsfragen werden zentrale Bestandteile kommunikativer Figurationen unter der Perspektive auf Familien im Distance Schooling in den Blick genommen: das familiale Medienensemble und die Akteurskonstellationen der Familie (Kammerl u. a. 2021). Neben den in der gesamten Figuration zur Verfügung stehenden Medien (Medienensemble) ist für eine Untersuchung des Distance Schooling insbesondere das Medienrepertoire des Kindes zu berücksichtigen. Für dessen Erfassung wurden die Kategorien «Medienrepertoire des Kindes» und «Medienpraktiken des Kindes» genutzt. Veränderungen im Medienensemble der Familie wurden anhand einer gleichnamigen Kategorie identifiziert.

Die zweite Forschungsfrage fokussiert das sich durch das Distance Schooling (potenziell) verändernde Beziehungsgefüge. Auch wenn auf Basis der Daten nicht das Beziehungsgeflecht der gesamten Familie beschrieben werden kann, lassen sich über die Dyade von interviewtem Elternteil und Kind wichtige Einblicke in die Struktur der Figuration Familie gewinnen. Dabei wurden für das Distance Schooling relevante Aushandlungsprozesse in den Themenbereichen Medienerziehung und familiale Lernorganisation untersucht. Mit der Kategorie «Medienerzieherische Praktiken» wurden hierzu mögliche Veränderungen der Medienregeln oder medienbezogene Konflikte erfasst. Das Konstrukt der familialen Lernorganisation, in dem sowohl die Organisation der Schularbeiten des Kindes selbst, als auch die Lernbegleitung und -kontrolle der Eltern berücksichtigt werden, wurde über verschiedene Unterkategorien erfasst, welche die Einstellungen und Wahrnehmungen von Kind und Elternteil bezüglich des Distance Schooling sowie lernbezogene oder unterstützende Medienpraktiken abbilden. Interviewaussagen, die eine Aushandlung in der Machtbalance zwischen Kind und Eltern(teil) erkennen liessen, wurden zusätzlich mit der Kategorie «Herstellung von Kontrolle/Autonomie» codiert.

\section{Darstellung der Ergebnisse}

\section{1 (Schulbezogene) Veränderungen der Medienensembles und -repertoires}

Hinsichtlich der Geräteanschaffungen erwiesen sich die Komponenten der familialen Medienensembles und der Medienrepertoires der Kinder in der ersten Phase des Distance Schooling als relativ stabil. Alle sechs Familien verfügten bereits über die für die Anforderungen der Schularbeiten notwendigen Geräte. Für den Austausch zwischen Eltern bzw. Kindern und Lehrkräften fungierte die E-Mail als zentrales Kommunikationsmedium. Zudem wurde vom Einsatz einer Internetplattform berichtet, die vom Kultusministerium betrieben wird. Über die Plattform und E-Mails wurden primär vorgegebene Arbeitsaufträge bereitgestellt und abgerufen. Eltern und Kinder berichteten gleichermassen, dass zu Beginn der Schulschliessungen die angebotene 
Plattform aufgrund von Überlastung kaum funktioniert habe. Fast alle Familien nutzten zum Abrufen der Arbeitsaufträge einen Computer bzw. Laptop, lediglich ein Kind verwendete hierfür sein Tablet und sein Smartphone. Lernangebote, die über den Versand von Arbeitsblättern hinausgingen (z. B. videobasierter Mathematik-Unterricht und Sprechstunden, Erklärvideos oder Lernaufträge via Apps) kamen zum Einsatz, wurden jedoch nur von einzelnen Lehrpersonen angeboten und blieben eine Ausnahme. In zwei Fällen (Hannah, Jakob) wurden Praktiken beschrieben, die sich die Kinder im Rahmen der Schularbeiten selbst angeeignet hatten (YouTube zur Eigenrecherche und Skype für die gemeinsame Bearbeitung von Schularbeiten).

Aufgrund des qualitativen Studiendesigns konnten die Verantwortung für die Bearbeitung der Schulaufgaben und die hierfür notwendigen Kompetenzen der Kinder genauer analysiert werden. Diese waren in den befragten Familien sehr unterschiedlich verteilt. So agierten einige Kinder recht eigenständig im Distance Schooling, indem sie sich beispielsweise selbst auf der Lernplattform registrierten, ihre Mails und Aufgaben alleine verwalteten oder persönlich mit den Lehrkräften kommunizierten. Andere Kinder wurden bei diesen Anforderungen dagegen stärker von ihren Eltern unterstützt. Bei diesen Kindern mussten die Eltern zum Beispiel häufig die Mails mit den Schulaufgaben verwalten.

Mit Blick auf das Medienrepertoire der Kinder zeigt sich in den Interviews, dass die Kinder in der Zeit des ersten Distance Schooling zunehmend gelernt haben, mit kleinen Schwierigkeiten umzugehen, (Bedien-)Kompetenzen erworben haben und entsprechend in der Lage waren eigenständiger zu agieren. ${ }^{3}$ Beispielsweise übernahm ein Junge (Jakob), nachdem zunächst seine Mutter die Mails der Schule organisiert und die Arbeitsblätter ausgedruckt hatte, diese Aufgaben sukzessive selbst. Die Ergebnisse bestätigen die skizzierten Befunde zur primär asynchronen Bereitstellung von Arbeitsaufträgen in der ersten Phase des Distance Schooling. Die neuen technischen Anforderungen wurden dabei innerhalb der Familie - durch die Kinder selbst und/oder unterstützend durch die Eltern - relativ problemlos bewältigt. Probleme mit dem Distance Schooling zeigten sich in erster Linie auf organisatorischer Ebene. Diese ergaben sich laut den befragten Eltern einerseits durch eine unstrukturierte Organisation durch Lehrkräfte und Schulen, andererseits durch die gestiegenen Anforderungen an die Kinder und Familien bezüglich der Lernorganisation.

\subsection{Familiale Aushandlungsprozesse im Kontext von Distance Schooling}

Die von den Eltern wahrgenommenen neuen Anforderungen durch das Distance Schooling bringt eine Mutter exemplarisch zum Ausdruck:

3 Eine Ausnahme stellt der Fall Johann dar. Hier erzählte die Mutter, dass aus ihrer Sicht die ersten Wochen im Distance Schooling gut liefen, ihre Kinder dann jedoch zunehmend Motivation und Eifer vermissen liessen (vgl. folgendes Kapitel). 
«Ich habe eigentlich das Gefühl, so, wie es jetzt im Augenblick läuft, wird diese Lerntätigkeit auf die Eltern abgewälzt.» (Mutter von Johann)

Diese plötzliche Veränderung des Alltags beinhaltete das Potenzial, die Rollen von Kindern und Eltern in der kommunikativen Figuration Familie hinsichtlich schulischen Lernens und des privaten Umgangs mit Medien in Frage zu stellen. Die familiale Lernorganisation stand in einem direkten Zusammenhang mit dem Distance Schooling, da die erste Phase der Schulschliessungen eine unmittelbare Veränderung der pädagogischen Rahmenbedingungen für das Kind implizierte. Aber auch hinsichtlich der Medienerziehung stellt sich die Frage, ob diese durch das Distance Schooling irritiert wurde, da Schultätigkeiten plötzlich an die Nutzung digitaler Medien geknüpft waren und sich gleichzeitig der Alltag der Kinder massiv veränderte. Mit Blick auf die autonomiebezogenen Aushandlungsprozesse lässt sich das Sample analytisch in zwei Gruppen typisieren. Die Gruppen wurden ausgehend von den beiden Themenbereichen medienerzieherische Praktiken und familiale Lernorganisation gebildet. In der einen Gruppe wurden Lernorganisation und Medienerziehung kaum modifiziert. In der anderen Gruppe waren diese beiden Themenbereiche Gegenstand von intensiveren Aushandlungsprozessen in der Akteurskonstellation zwischen Kind und Elternteil.

Geringere Aushandlungsprozesse: Herausforderungen werden im bestehenden Beziehungsgeflecht aufgefangen

In den drei Fällen (Pia, Hannah, Tobias), in denen durch das Distance Schooling die familiale Lernorganisation und die Medienerziehung kaum modifiziert wurden, spielten autonomiebezogene Aushandlungsprozesse eine geringe Rolle. Das figurative Interdependenzgeflecht blieb bei diesen Familien hinsichtlich der familialen Lernorganisation und der Medienerziehung weitgehend konstant, wenngleich beide Bereiche in den Familien unterschiedlich ausgestaltet wurden. Dieser Umstand wird im Folgenden an den drei Fällen der Gruppe diskutiert.

Hinsichtlich einer durch das Distance Schooling veränderten Lernorganisation berichtete die Mutter von Pia, dass ihre Tochter immer «brav» ihre Arbeiten erledigen würde. Dabei zeigte sich aber auch, dass die Mutter die Bearbeitung der Schularbeiten vollständig kontrollierte. Anfangs habe sie ihrer Tochter die Schularbeiten als «Übung» zur Selbstständigkeit alleine überlassen, in den folgenden Wochen wollte sie die Lernbegleitung aber wieder intensivieren, da dann auch neuer Unterrichtsstoff behandelt werde. Die Entscheidungen über die Lernorganisation traf alleine die Mutter, in Pias Aussagen liessen sich keine Autonomiebestrebungen ausmachen. Somit wurden im Rahmen der etablierten Machtbalance zwischen Pia und ihrer Mutter Routinen im Distance Schooling gefunden. In einem anderen Fall (Hannah) wurden von der Mutter ebenfalls kleinere Schwierigkeiten bezüglich des selbstständigen 
Arbeitens ihrer Tochter sowie ein fehlender schulischer Druck wahrgenommen, diese aber als weniger problematisch gesehen. Über die Organisation der E-Mails griff die Mutter unterstützend in das Distance Schooling ein und sah sich mehr in die Schularbeiten eingebunden, da sie Hannah manchmal etwas erklären oder für sie recherchieren musste. Die konkrete Bearbeitung und Einteilung der Aufgaben überliess sie jedoch Hannah selbst. Trotz der wahrgenommenen Probleme traten keine Konflikte zwischen Hannah und ihrer Mutter hinsichtlich des Distance Schooling auf - auch hier erwiesen sich die Machtbalancen relativ stabil.

Mit Blick auf die Medienerziehung wiesen die beiden Fälle die Gemeinsamkeit auf, dass in den Familien keine festgelegten medienbezogenen Regeln vorherrschten und die Eltern eher situationsgebunden auf den Medienkonsum der Kinder Einfluss nahmen:

«Aber wir haben nie wirklich Regeln gehabt. Also, also wenn es halt zu viel ist, dann muss man Pause machen und, ja. Und ähm (.) ja, wahrscheinlich würde ich momentan schon eher mehr durchgehen lassen, weil es ist ja einfach mehr Zeit da.» (Mutter von Pia)

Pia selbst erwähnte nicht, dass sie bestimmte Medienangebote in der Phase der Schulschliessung öfter nutzen durfte. Ähnlich wie bei den Schularbeiten strukturierte die Mutter auch das Medienrepertoire ihrer Tochter, wobei sich auch hier seitens Pia keine Autonomiebestrebungen ausmachen liessen. Ähnliches gilt im Fall von Hannah, wobei hier die Mutter das Medienrepertoire und die Nutzungszeiten der Tochter eher im Stile eines «Laissez-faire» begleitet, da ihrer Ansicht nach Hannah einen guten eigenverantwortlichen Umgang mit ihrem Medienkonsum zeigte.

Auch im dritten Fall (Tobias) liessen sich keine bedeutsamen autonomiebezogenen Aushandlungen bei der familialen Lernorganisation beobachten. Tobias agierte von Beginn an sehr eigenständig im Distance Schooling. Er kommunizierte direkt mit den Lehrkräften und war mit seiner eigenen Mailadresse auf der angebotenen Lernplattform registriert. Bei den Aufgabenplänen, die in Folge von Schwierigkeiten zu Beginn des Distance Schooling etabliert wurden, unterstützte inn zwar anfangs seine Mutter, zum Erhebungszeitpunkt erstellte Tobias diese jedoch alleine. Sowohl der Vater als auch Tobias sprachen vor allem über entlastende Aspekte durch das Distance Schooling, z. B. dass man mit geringem Zeitaufwand ein höheres Lernpensum bewältigen könne. Medienerzieherische Praktiken wurden im Fall Tobias nicht durch die Umstände tangiert. ${ }^{4}$

In den drei Fällen liess sich zusammenfassend beobachten, dass Anpassungen an die Bedingungen des Distance Schooling im bestehenden Beziehungsgeflecht und ohne autonomiebezogene Aushandlungsprozesse verliefen. Die Veränderungen

4 Tobias berichtete, dass er einige Wochen vor den Schulschliessungen mit seinen Eltern eine Ausweitung seiner regulierten Computerspielzeit vereinbart hatte. Seitdem traten (auch im Distance Schooling) keine medienbezogenen Konflikte auf. 
in der familialen Lernorganisation etablierten sich ohne Konflikte, trotz des veränderten Alltags wurden keine medienbezogenen Regularien tangiert. In beiden Themenbereichen agierten die Kinder unterschiedlich selbstständig, das Verhältnis von Kontrolle und Autonomie in der Beziehung zwischen Kind und Elternteil blieb dabei relativ konstant.

Intensivere Aushandlungsprozesse: Veränderung des Verhältnisses von Kontrolle und Autonomie

In den drei verbleibenden Fällen des Samples (Jakob, Lisa, Johann) zeigten sich intensivere autonomiebezogene Aushandlungsprozesse, die mit einer Veränderung der familialen Lernorganisation und der Medienerziehung in Zusammenhang stehen. In diesen Fällen liess sich beobachten, wie die Beziehung zwischen Eltern(teil) und Kind insbesondere mit Blick auf die Selbstständigkeit des Kindes herausgefordert wurde.

In Bezug auf die familiale Lernorganisation sprachen die interviewten Mütter in zwei Fällen (Jakob und Lisa) davon, dass gerade zu Beginn der Schulschliessungsphase die Organisation des Distance Schooling «chaotisch» verlief und mit Problemen behaftet war. Zum Zeitpunkt der Interviews hätten sich aber bereits funktionierende Abläufe und Routinen eingespielt. Bei Jakob war dieser Prozess mit einem Autonomiegewinn bzw. einer vertrauensbasierten Autonomiegewährung der Mutter verbunden. Sie berichtete, dass Jakob sein Lernen inzwischen selbstständig organisiere und sie dies kaum mehr mitbekomme. Gleichzeitig schilderte sie den Eindruck, dass ihr Sohn alles erledigen und viel schaffen würde. Beispielsweise wäre er auch selbst auf die Idee gekommen, die Schularbeiten mit einem Freund gemeinsam via Skype zu bearbeiten.

Bei Lisa waren anfängliche Probleme der Mutter zufolge damit verbunden, dass ihre Tochter erst begreifen musste, dass auch Distance Schooling «echte Schule» sei. Auch wenn die Mutter ihre Tochter als sehr selbstständig einschätzte, berichtete sie, dass Lisa bei den Schulaufgaben im Distance Schooling gerne auch mal «schludern» würde. Die Mutter hatte deshalb den Eindruck, dass die Unterstützung von Lisa unter diesen Bedingungen zeitaufwendiger sei. Allerdings fiel es ihr schwer, den Unterstützungsaufwand richtig einzuschätzen, da sie nicht wisse, ob Lisa ein Thema nicht verstehe oder sich zu unkonzentriert damit auseinandersetze. Anfangs hätte die Mutter abwechselnd mit ihrem Partner alle 30 Minuten nach ihrer Tochter schauen müssen, um zu sehen, ob sie sich mit ihren Schularbeiten beschäftigt. Mit der Zeit habe sie aber nur noch alle zwei Stunden nachgefragt, ob hinsichtlich der Schularbeiten alles in Ordnung sei. Lisa hätte ausserdem schnell selbst gemerkt, dass es besser sei, die Aufgaben gleich vormittags zu erledigen, statt den ganzen Tag aufzuschieben. Von einem Wissensdefizit berichtete die Mutter hinsichtlich der Aufgaben, die ihre Tochter 
direkt am PC bearbeitet und absendet. Hier wisse sie über den Inhalt nicht Bescheid. Insgesamt freute sich die Mutter darauf, wenn das Thema Schule für sie bald wieder mehr in den Hintergrund rücken würde.

Jakob geniesst im Vergleich zu Lisa und Johann bei seiner Lernorganisation zwar ein hohes Vertrauen seitens seiner Eltern, in der Medienerziehung wird Jakob aber deutlicher kontrolliert und reglementiert. Dabei nutzen die Eltern die App «FamilyLink» zur technischen Festlegung von Zeitlimits an Jakobs Handy und Tablet und um seine Aktivitäten einzusehen. Im Zuge des Distance Schooling zeigten sich die Eltern etwas flexibler und verlängerten gelegentlich Jakobs Zeitlimit. Seine Mutter sprach davon, dass ein bis zweimal in der Woche eine solche Ausnahme vorkomme. Sie betonte, das Zeitlimit nur dann zu erhöhen, wenn sie sehe, dass Jakob die Medienzeit primär für schulbezogene Aktivitäten oder für den Kontakt zu Peers genutzt habe. Jakob selbst erlebte seine Eltern in der Zeit des Distance Schooling als weniger streng bezüglich seiner Mediennutzung. Bei Lisa zeigte sich dagegen ein anderes Bild. Die Eltern wollten die bestehenden Regeln zur Mediennutzungszeit anfänglich aufrechterhalten. Dies habe allerdings nicht funktioniert und zu Konflikten geführt. Nach ein paar Wochen wäre dann bei beiden die Überlegung gereift, die regulierte Medienzeit für Lisa flexibler zu gestalten. So stellte Lisa auf die Frage, was für sie die grösste Veränderung seit der Schulschliessung sei, fest: «Man kann den ganzen Tag am Handy sein».

Bei einer ersten abstrahierenden Betrachtung der beiden Fälle zeigt sich, dass sich die Struktur der kommunikativen Figuration im Kontext der familialen Lernorganisation und der Medienerziehung verändert hat. Bei der Medienerziehung wurden bestehende Reglementierungen gelockert. Während diese Regelanpassungen bei Lisa eine Folge vorausgegangener Konflikte waren, gewährten Jakobs Eltern ihrem Sohn umfangreichere Medienzeiten. Ein Autonomiegewinn bezüglich der Lernorganisation kann bei beiden Kindern beobachtet werden. Nachdem die Kinder eigenständiger ihre Aufgaben im Distance Schooling organisierten, reduzierten die Eltern ihre Unterstützung (Jakob) bzw. Kontrolle (Lisa) beim Lernen zuhause. Deutlich wird dabei, dass die Themenfelder der familialen Lernorganisation und der Medienerziehung - auch wenn sie stellenweise getrennt verhandelt werden - miteinander zusammenhängen. Dies betrifft die Strukturierung des Medienrepertoires des Kindes durch Regeln der Eltern sowie die Akteurskonstellation zwischen Kind und Elternteil, die sich durch die Situation im Distance Schooling veränderten. In beiden Themenfeldern eröffnen sich für die Kinder potenziell neue Freiheitsgrade, die in den Familien in unterschiedlichem Umfang ausgehandelt wurden.

Diese ersten Überlegungen lassen sich auch im dritten Fall der vorliegenden Gruppe wiederfinden. Bei Johann zeigt sich eine stark kontrollierende und reglementierende Position der Mutter, die sich vor allem in der Lernorganisation widerspiegelt. Durch die Irritationen des Distance Schooling kommt es in der Familie zu 
deutlichen Anpassungen der zuvor routinierten Kontrolle und Unterstützung der Schularbeiten. Vor den ersten Schulschliessungen spielte die Mutter eine wichtige Rolle in der Lernorganisation von Johann. Während der andauernden Schulschliessungen wurde es für sie notwendig, diese Routine unter Einbezug des Vaters zu ändern. Von dem restlichen Sample unterscheidet sich der Fall Johann zudem darin, dass das Distance Schooling nach Aussage der Mutter zu Beginn der Schulschliessungen besser funktioniert habe:

«Das hat die ersten zwei Wochen, wo das neu war, (.) hat das ganz gut geklappt. Also da hatten wir diese Arbeitsaufträge. Da hat man das den Kindern gegeben und dann haben sie da auch gearbeitet, aber nach zwei Wochen war dann die Luft raus.» (Mutter von Johann)

Mehrfach äusserte die Mutter im Interview das Problem, dass Johann nicht selbstständig und gewissenhaft seine Aufgaben erledigen würde. Dadurch sah sie sich durchweg in der Pflicht, nach ihrem Feierabend im Homeoffice die Schulaufgaben nachzuarbeiten. Ihr fehle ein «Patentrezept», wie sie ihre Kinder zu einer eigenständigen Aufgabenbearbeitung motivieren könne. Zwar rief Johann selbstständig seine Aufgaben für die Schule ab, jedoch in erster Linie, weil inn seine Mutter dazu verpflichte. Gleichzeitig fehlte ihr das Vertrauen in Johanns Eigenverantwortlichkeit: «Und das ruft er selber ab. (.) Da sind wir auch nicht daneben und/ und (.) machen irgendwas, ne. Sondern, da erwarte ich auch von ihm, dass er es eigenständig macht (.) Ob er es macht, werden wir sehen.» (Mutter von Johann)

Dass Johann von den meisten seiner Lehrkräfte neben den Aufgaben zugleich auch die Lösungen zur Verfügung gestellt bekam, forderte die Frage nach Verantwortlichkeiten bei der Schularbeit zusätzlich heraus. Die Mutter meinte diesbezüglich, dass sie nicht die ganze Zeit kontrollieren würde, ob Johann sich erst die Lösungen anschaut. Sie äusserte darüber hinaus - wie einige andere Kinder und Eltern auch - den Wunsch nach einem videobasierten Unterricht zu etablierten Zeiten, betonte aber in erster Linie den verbindlichen und prüfenden Charakter von Unterrichtsstunden. Im Rahmen der Medienerziehung wurden im Fall Johann die Medienregeln stark gelockert, primär um Johann unter den gegebenen Umständen beschäftigt zu wissen und Zeit für die eigenen beruflichen Aufgaben zu haben. Dies betraf die Aufhebung der limitierten Nutzungszeit auf Johanns Smartphone. Im Gegensatz zu den anderen Fällen deutet sich bei Johann im Verlauf des Distance Schooling ein Verlust von Autonomie in der Lernorganisation an. Andererseits wurde ihm bezüglich seiner Mediennutzung, ähnlich wie bei Lisa, aufgrund der Alltagsumstände mehr Autonomie zugestanden. 
In den drei skizzierten Familien liess sich im Zuge der Bewältigung der neuen Umstände durch Distance Schooling eine qualitative Veränderung in der Akteurskonstellation zwischen Kind und Eltern(teil) beobachten. Die Veränderungen der familialen Lernorganisation und/oder medienerzieherischer Praktiken gehen - wenngleich auf unterschiedliche Weise - mit autonomiebezogenen Aushandlungsprozessen einher. Die differente Wahrnehmung der ersten Phase des Distance Schooling durch die Familien kann somit im Zusammenhang mit der Aushandlung innerfamilialer Beziehungen betrachtet werden.

\section{Fazit und Diskussion}

Die dargestellten Ergebnisse zeigen, dass der Umbruch zum Distance Schooling in der Phase der ersten Schulschliessungen die kommunikative Figuration Familie in unterschiedlicher Weise herausfordert. Die Gestaltung des Distance Schooling hat u. a. einen Einfluss auf das Verhältnis von kindlicher Autonomie und Heteronomie. In den Aushandlungsprozessen im Kontext des Distance Schooling überlagern sich zwei Themenbereiche. Da für die Bearbeitung der Schulaufgaben die Nutzung digitaler Medien erforderlich ist, begrenzt sich die Aushandlung der kindlichen Autonomie nicht auf die familiale Lernorganisation zur Bearbeitung der Schulaufgaben. Auch die kindliche Mediennutzung und somit medienerzieherische Fragestellungen werden durch die Schulschliessungen tangiert. In dem Sample zeigt sich - trotz seiner sozioökonomisch homogenen Ausrichtung - eine deutliche Heterogenität hinsichtlich der autonomiebezogenen Aushandlungsprozesse in beiden Themenbereichen. Die heterogenen Aushandlungsprozesse liessen sich idealtypisch gruppieren. Bei der einen Hälfte des Samples erfolgte die familiale Lernorganisation und die Medienerziehung konfliktfrei im durch die Eltern vorgegebenen Rahmen. Bei der anderen Hälfte zeigten sich hingegen vermehrt Konstellationen, in denen die familiale Lernorganisation und/oder die Bedingungen der Medienerziehung zu - teilweise konflikthaften Aushandlungsprozessen führten. Einflussfaktoren, die diese unterschiedlichen Aushandlungsprozesse bedingen, konnten in der explorativen Studie nicht identifiziert werden.

Stattdessen wird aus einer figurationsanalytischen Perspektive die Relevanz des familialen Beziehungsgeflechts für diese Aushandlungsprozesse deutlich. So stehen immer strukturierende und autonomiebezogene Prozesse in Wechselwirkung zueinander. Einerseits beziehen sich strukturierende - beispielsweise medienerzieherische - Praktiken der Eltern immer auf die stattfindenden Handlungspraktiken und somit auf die vorhandenen Kompetenzen der Kinder. Insbesondere zu Beginn des Distance Schooling wurden dementsprechend unterstützende und strukturierende Massnahmen erforderlich, wenn Kinder nicht über die notwendigen Kompetenzen zur Bewältigung der Anforderungen verfügten. Andererseits sind auch die kindlichen 
Autonomiebestrebungen immer auf die durch die Eltern vorgegebenen Rahmenbedingungen bezogen. Liegen beispielsweise - wie im Fall von Johann - stark strukturierende und reglementierende Rahmenbedingungen vor, können sich die kindlichen Autonomiebestrebungen auch nur auf diese Gegebenheiten beziehen. In den familialen Aushandlungsprozessen bezüglich der kindlichen Autonomie liessen sich somit fluktuierende Machtbalancen identifizieren, die in Bezug zu dem Thema Distance Schooling stehen.

Aus (medien-)pädagogischer Sicht sind die Befunde hinsichtlich der im Distance Schooling erforderlichen und erworbenen Kompetenzen interessant. In den Interviews zeigte sich, dass die eigenständige schulbezogene und persönliche Mediennutzung der Kinder an innerfamiliale Aushandlungsprozesse gebunden ist. Der Kompetenzerwerb der Kinder ist somit auch davon abhängig, ob das Verhältnis von Strukturierung und Eigenständigkeit für das Kind so gestaltet wird, dass es mit der Ausgangslage des Kindes übereinstimmt. Gerade die eigenständige Mediennutzung (zur Bearbeitung von schulischen Lernaufgaben) erhält hierbei eine besondere Bedeutung. So wird die Aneignung von Selbstlern- und Medienkompetenzen als zentrale Zieldimension für Kinder im Distance Schooling betrachtet (Gerhardts u. a. 2020b). Die Analyse des familialen figurativen Interdependenzgeflechts und der Aushandlung von Autonomie und Heteronomie kann dazu beitragen, familiale und medienbezogene Lernkontexte von Kindern in dieser besonderen Situation besser zu verstehen. Gerade bei der Frage nach der Entwicklung und Förderung von Lern- und Medienkompetenzen im Distance Schooling erscheint es deshalb zielführend, die Rolle des familialen Beziehungsgeflechts - zum Beispiel über den Ansatz kommunikativer Figurationen - in die Betrachtung miteinzubeziehen.

Die dargestellten Befunde der vorliegenden sowie vieler anderer inzwischen publizierter Studien berücksichtigen primär die erste Phase des Distance Schooling im Zuge der Schulschliessungen im Frühjahr 2020. Einige familiale Aushandlungsprozesse ergeben sich womöglich durch die für diesen Zeitraum konstitutiven Umstände (z. B. primär asynchrone Lehrformen), die sich im weiteren Verlauf der Covid-19-Pandemie verändert haben. Aufgrund des andauernden und dynamischen Verlaufs der Pandemie verfügen Lehrkräfte, Eltern sowie Schülerinnen und Schüler inzwischen über Erfahrungen im Umgang mit einem eingeschränkten Präsenzunterricht. Um auch langfristige Transformationsprozesse in der Figuration der Familie, die über die abrupte Ausnahmesituation im Frühjahr 2020 hinausgehen, betrachten zu können, sind weitere Erhebungen desiderabel. Dabei gilt es zu beleuchten, wie sich die Gestaltung und Organisation des Distance Schooling - auch als den Präsenzunterricht ergänzende Unterrichtsform - entwickelt (hat) und wie diese Entwicklungen im familialen Interdependenzgeflecht bearbeitet werden. 
Die explorative Studie zeigt, dass das Konzept kommunikativer Figurationen ein adäquates, heuristisches Instrument darstellt, die familialen Bedingungen zu erforschen, in denen Distance Schooling stattfindet. Eine Aufgabe weiterführender Forschung besteht darin, das familiale Interdependenzgeflecht umfassender zu analysieren, wobei insbesondere die Perspektiven weiterer Familienmitglieder, wie die des zweiten Elternteils und möglicher Geschwister, mit einbezogen werden sollten. Ausserdem sollte in weiterführenden Erhebungen das Sample erweitert und hierdurch dessen sozioökonomische Verzerrung reduziert werden.

Die dargestellten Ergebnisse ergänzen vorhandene Studien zum Distance Schooling, indem sie Veränderungen der familialen Interaktionsprozesse durch die Schulschliessungen beleuchten. Bei dieser Betrachtung rückt die Frage in den Vordergrund, welche Bedingungen eine möglichst eigenständige Mediennutzung ermöglichen. Die Befunde zeigen, dass eigenständiges Medienhandeln immer auch auf - teilweise durchaus konfliktbehafteten - Aushandlungsprozessen basiert. Dabei wird deutlich, mit welchen Herausforderungen die (Ausnahme-)Situation des Distance Schooling verbunden ist und wie unterschiedlich Familien mit diesen umgehen. In der familialen Interaktion ergeben sich zugleich neue Freiheitsgrade und Anforderungen an ein eigenständiges Handeln der Kinder, die innerhalb der Familie ausgehandelt und bewältigt werden müssen.

\section{Literatur}

Andresen, Sabine, Anna Lips, Renate Möller, Tanja Rusack, Wolfgang Schröer, Severine Thomas, und Johanna Wilmes. 2020. «Kinder, Eltern und ihre Erfahrungen während der CoronaPandemie. Erste Ergebnisse der bundesweiten Studie KiCo». https://doi.org/10.18442/121.

BPA, Presse- und Informationsamt der Bundesregierung. 2020a. «Vereinbarung zwischen der Bundesregierung und den Regierungschefinnen und Regierungschefs der Bundesländer angesichts der Corona-Epidemie in Deutschland». Pressemitteilung 96. https://www.bundesregierung.de/breg-de/aktuelles/vereinbarung-zwischen-der-bundesregierung-undden-regierungschefinnen-und-regierungschefs-der-bundeslaender-angesichts-der-corona-epidemie-in-deutschland-1730934.

BPA, Presse- und Informationsamt der Bundesregierung. 2020b. «Besprechung von Bundeskanzlerin Merkel mit den Regierungschefinnen und Regierungschefs der Länder zum Coronavirus». Pressemitteilung 104. https://www.bundesregierung.de/breg-de/themen/coronavirus/besprechung-von-bundeskanzlerin-merkel-mit-den-regierungschefinnen-undregierungschefs-der-laender-zum-coronavirus-1733266.

Elias, Norbert. 1970. Was ist Soziologie? Grundfragen der Soziologie. Weinheim: Juventa. 
Fickermann, Detlef, und Benjamin Edelstein. 2020. "Editorial». In 〈Langsam vermisse ich die Schule ...). Schule während und nach der Corona-Pandemie., herausgegeben von Detlef Fickermann und Benjamin Edelstein, DDS Die Deutsche Schule Beiheft, Band 16:9-33. Waxmann. https://doi.org/10.31244/9783830992318.01.

Gerhardts, Lara, Anna-Maria Kamin, Dorothee M. Meister, Lea Richter, und Jeannine Teichert. 2020a. «Lernen auf Distanz - Einblicke in den familialen Alltag des Homeschoolings und Formen der Bewältigung». Medienimpulse, Juni, 26. https://doi.org/10.21243/MI-02-20-30.

Gerhardts, Lara, Anna-Maria Kamin, Dorothee M. Meister, Lea Richter, und Jeannine Teichert. 2020b. "Entwicklung von Selbstlern- und Medienkompetenz im Homeschooling - Chancen und konzeptionelle Anforderungen». PraxisForschungLehrer*innenBildung. Zeitschrift für Schul- und Professionsentwicklung. Vol. 2 No. 6: 139-54. https://doi.org/10.4119/PFLB3909.

Hasebrink, Uwe. 2014. «Die kommunikative Figuration von Familien: Medien, Kommunikation und Informationstechnologie im Familienalltag». In Die Zukunft der Familie. Anforderungen an Familienpolitik und Familienwissenschaft. Tagungsband zum 4. Europäischen Fachkongress Familienforschung, herausgegeben von Marina Rupp, Olaf Kapella, und Norbert F. Schneider, 225-39. Opladen: Barbara Budrich. https://doi.org/10.2307/j.ctvdf01kz.18.

Heller, Sonja, und Oliver Zügel. 2020. «Schule zu Hause» in Deutschland. Bestandsaufnahme im Corona-Lockdown aus Perspektive der Schüler-/innen und Eltern». Deutsche Telekom Stiftung. https://www.telekom-stiftung.de/sites/default/files/files/media/publications/ Ergebnisbericht-Homeschooling.pdf.

Hepp, Andreas. 2020. Deep mediatization. Key ideas in media and cultural studies. London: Routledge.

Hepp, Andreas, und Uwe Hasebrink. 2014. «Kommunikative Figurationen. Ein Ansatz zur Analyse der Transformation mediatisierter Gesellschaften und Kulturen». In Von der GutenbergGalaxis zur Google-Galaxis. Alte und neue Grenzvermessungen nach 50 Jahren DGPuK, herausgegeben von Birgit Stark, Oliver Quiring, Nikolaus Jackob, und Deutsche Gesellschaft für Publizistik- und Kommunikationswissenschaft, 343-60. Schriftenreihe der Deutschen Gesellschaft für Publizistik- und Kommunikationswissenschaft 41. Konstanz: UVK.

Hepp, Andreas, und Uwe Hasebrink. 2017. «Kommunikative Figurationen. Ein konzeptioneller Rahmen zur Erforschung kommunikativer Konstruktionsprozesse in Zeiten tiefgreifender Mediatisierung». Medien \& Kommunikationswissenschaft 65 (2): 330-47. https://doi. org/10.5771/1615-634X-2017-2-330.

Huber, Stephan Gerhard, Paula Sophie Günther, Nadine Schneider, Christoph Helm, Maruis Schwander, Julia Schneider, und Jane Pruitt. 2020. COVID-19 und aktuelle Herausforderungen in Schule und Bildung: erste Befunde des Schul-Barometers in Deutschland, Österreich und der Schweiz. https://doabooks.org/doab?func=fulltext\&uiLanguage=en\&rid=45543.

JIMplus. 2020. «JIMplus 2020 - Lernen und Freizeit in der Corona-Krise». mpfs. Medienpädagogischer Forschungsverbund Südwest. https://www.mpfs.de/fileadmin/files/Studien/JIM/ JIMplus_2020/JIMplus_2020_Corona.pdf. 
Kammerl, Rudolf, Claudia Lampert, Jane Müller, Marcel Rechlitz, und Katrin Potzel. 2021. «Mediatisierte Sozialisationsprozesse erforschen. Methodologische Implikationen». In Jahrbuch Medienpädagogik 16. Medienpädagogik in Zeiten tiefgreifender Mediatisierung, herausgegeben von Karsten Wolf, Sandra Aßmann, Patrick Bettinger, und Klaus Rummler. https://doi.org/10.21240/mpaed/jb16/2021.02.24.X.

Kammerl, Rudolf, Jane Müller, Claudia Lampert, Marcel Rechlitz, und Katrin Potzel. 2020. «Kommunikative Figurationen. Ein theoretisches Konzept zur Beschreibung von Sozialisationsprozessen und deren Wandel in mediatisierten Gesellschaften?». In Bewegungen. Beiträge zum 26. Kongress der Deutschen Gesellschaft für Erzieungswissenschaft, herausgegeben von Isabell van Ackeren, Helmut Bremer, Fabian Kessl, Hans Christoph Koller, Nicolle Pfaff, Caroline Rotter, Dominique Klein, und Ulrich Salaschek, 377-88. Barbara Budrich. https://doi.org/10.2307/j.ctv10h9fjc.30.

Kuckartz, Udo. 2020. Einführung in die computergestützte Analyse qualitativer Daten. 4. Aufl. Wiesbaden: Springer VS.

Lampert, Claudia, Kira Thiel, und Begüm Güngör. 2021. «Mediennutzung und Schule zur Zeit des ersten Lockdowns während der Covid-19-Pandemie 2020: Ergebnisse einer Online-Befragung von 10- bis 18-Jährigen in Deutschland». Arbeitspapiere des Hans-Bredow-Instituts. https://doi.org/10.21241/SSOAR.71712.

Langmeyer, Alexandra, Angelika Guglhör-Rudan, Thorsten Naab, Marc Urlen, und Ursula Winklhofer. 2020. «Kindsein in Zeiten von Corona. Erste Ergebnisse zum veränderten Alltag und zum Wohlbefinden von Kindern». Deutsches Jugendinstitut. https://www.dji.de/ fileadmin/user_upload/dasdji/themen/Familie/DJI_Kindsein_Corona_Erste_Ergebnisse. pdf.

Möhring, Katja, Elias Naumann, Maximiliane Reifenscheid, Annelies G. Blom, Alexander Wenz, Tobais Rettig, Roni Lehrer, u. a. 2020. «Die Mannheimer Corona-Studie: Schwerpunktbericht zu Erwerbstätigkeit und Kinderbetreuung». https://www.uni-mannheim.de/media/ Einrichtungen/gip/Corona_Studie/2020-04-05_Schwerpunktbericht_Erwerbstaetigkeit_ und_Kinderbetreuung.pdf.

Robert-Bosch-Stiftung. 2020. «Das Deutsche Schulbarometer Spezial Corona-Krise. Ergebnisse einer Befragung von Lehrerinnen und Lehrern an allgemeinbildenden Schulen im Auftrag der Robert Bosch Stiftung in Kooperation mit der ZEIT». forsa Politik- und Sozialforschung GmbH. https://www.schulwerk-bayern.de/fileadmin/pics/Aktuelles_2020/20-04-27_Schulentwicklung_Forsat.pdf.

Unger, Valentin, Yoka Krämer, und Albrecht Wacker. 2020. «Unterricht während der CoronaPandemie». PraxisForschungLehrer*innenBildung. Zeitschrift für Schul- und Professionsentwicklung. Vol. 2 No. 6: 84-99. https://doi.org/10.4119/PFLB-3907.

Vodafone Stiftung. 2020. «Unter Druck. Die Situation von Eltern und ihren schulpflichtigen Kindern während der Schulschließungen». Eine Befragung der Vodafone Stiftung Deutschland. Vodafone Stiftung Deutschland gGmbH. http://docs.dpaq.de/16200-studie_vsd_elternbefragung.pdf. 
Wacker, Albrecht, Valentin Unger, und Thomas Rey. 2020. ",Sind doch Corona-Ferien, oder nicht?". Befunde einer Schüler*innenbefragung zum 〈Fernunterricht»». In „Langsam vermisse ich die Schule ...", herausgegeben von Detlef Fickermann und Benjamin Edelstein, 79-94. Waxmann. https://doi.org/10.31244/9783830992318.04.

Wildemann, Anja, und Ingmar Hosenfeld. 2020. «Bundesweite Elternbefragung zu Homeschooling während der Covid 19-Pandemie. Erkenntnisse zur Umsetzung des Homeschoolings in Deutschland». Institut für Bildung im Kindes- und Jugendalter \& Zentrum für Empirische pädagogische Forschung. Universität Koblenz-Landau. http://www.zepf.eu/wp-content/ uploads/2020/06/Bericht_HOMEschooling2020.pdf.

Wößmann, Ludger, Vera Freundl, Elisabeth Grewenig, Philipp Lergetporer, Katharina Werner, und Larissa Zierow. 2020. «Bildung in der Coronakrise: Wie haben die Schulkinder die Zeit der Schulschließungen verbracht, und welche Bildungsmaßnahmen befürworten die Deutschen?» ifo Schnelldienst, 2020, 73, Nr. 09, 01-17. München: ifo Institut. https://www.ifo. de/en/publikationen/2020/article-journal/bildung-der-coronakrise-wie-haben-die-schulkinder-die-zeit-der. 